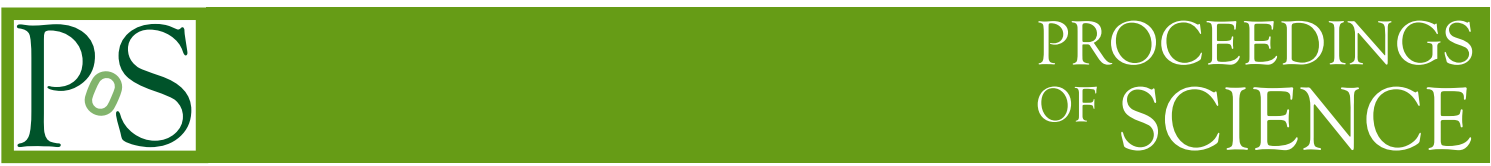

\title{
The Wave Function of the Roper Resonance
}

\section{Dale S. Roberts*}

Special Research Centre for the Subatomic Structure of Matter, School of Chemistry \& Physics, University of Adelaide, SA, 5005, Australia

\section{Waseem Kamleh}

Special Research Centre for the Subatomic Structure of Matter, School of Chemistry \& Physics, University of Adelaide, SA, 5005, Australia

\section{Derek B. Leinweber}

Special Research Centre for the Subatomic Structure of Matter, School of Chemistry \& Physics, University of Adelaide, SA, 5005, Australia

\begin{abstract}
We apply the eigenvectors from a variational analysis to successfully extract the wave function of the Roper state, and the next $P_{11}$ state of the nucleon, associated with the $N^{\star}(1710)$. We find that both states exhibit a structure consistent with a constituent quark model. The Roper d-quark wave function contains a single node consistent with a $2 S$ state, and the $N^{\star}(1710)$ contains two, consistent with a $3 S$ state. A detailed investigation of the mass dependence of the wave functions of these states is carried out at four quark masses. The lightest mass provides a pion mass of 156 $\mathrm{MeV}$, just slightly above the physical mass of $139.6 \mathrm{MeV}$. The ground state wave function shows little mass dependence, consistent with prior wave function investigations. The wave functions of the Roper and second excited state show finite volume effects which become prominent at heavier quark masses than for the ground state.
\end{abstract}

The 30 International Symposium on Lattice Field Theory - Lattice 2012,

June 24-29, 2012

Cairns, Australia

${ }^{*}$ Speaker. 
Understanding the mass dependence of states in lattice QCD has been a necessary part of calculations since the inception of lattice gauge theory. Though modern simulations are capable of accessing the physical quark mass regime, it is still important that the mass dependence be understood, especially as excited state studies progress.

Visualizations of the probability distribution on the lattice have been used to observe interesting physical effects such as Lorentz contraction [1, 2], quarks aligning with a magnetic field and diquark clustering [3]. Furthermore, the probability distribution can be used as a diagnostic tool, allowing finite volume effects and other lattice artifacts to be easily visualized and understood as the quark mass changes.

Robust methods have been developed that allow the isolation and study of states associated with these resonances in Lattice QCD [4, 5, 6, 7, 8, 9, 10, 11]. In this study, we apply the variational method $[12,13,14]$ to extract the ground state and first two $P_{11}$ excited states of the proton, the Roper [15] and the $N^{\star}(1710)$. We will then combine this with lattice wave function techniques to calculate the probability distributions of these states at a variety of quark masses.

The wave function of a hadron is proportional to the two point Green's function,

$$
G_{i j}(\vec{p}, t)=\sum_{x} e^{-i \vec{p} \cdot \vec{x}}\left\langle\Omega\left|T\left\{\chi_{i}(\vec{x}) \bar{\chi}_{j}(0)\right\}\right| \Omega\right\rangle,
$$

where $\chi_{i}$ are the standard proton interpolating fields. In order to construct the wave function, the quark fields in the annihilation operator are each given a spatial dependence,

$$
\chi_{1}(\vec{x}, \vec{y}, \vec{z}, \vec{w})=\varepsilon^{a b c}\left(u_{a}^{T}(\vec{x}+\vec{y}) C \gamma_{5} d_{b}(\vec{x}+\vec{z})\right) u_{c}(\vec{x}+\vec{w}),
$$

while the creation operator remains local. This generalizes $G(\vec{p}, t)$ to a wave function proportional to $G(\vec{p}, t ; \vec{y}, \vec{z}, \vec{w})$. In principle, we could allow each of these coordinates, $\vec{y}, \vec{z}, \vec{w}$, to vary across the entire lattice, however, we can reduce the complexity by taking advantage of the hyper-cubic rotational and translational symmetries of the lattice. A description of the probability distribution of a particular quark within the proton can be formed by holding two of the quarks at fixed spatial points and calculating the third quark's wave function for every lattice site. For this study, we will focus on the probability distribution of the $d$ quark from Eq. (2) with the $u$-quarks fixed at the origin. The resulting construct is gauge dependent so we choose to fix the gauge configurations to Landau gauge.

Different interpolators exhibit different couplings to the proton ground and excited states and hence can be used to construct a variational basis. The limited number of local interpolators restricts the size of the operator basis [4]. To remedy this, one can exploit the smearing dependence of the coupling of states to one or more standard interpolating operators in order to construct a larger variational basis where the $\chi_{i}$ and $\bar{\chi}_{j}$ from Eq. (1) contain a smearing dependence. This method has been shown to allow access to states associated with resonances such as the Roper, $N^{\star}(1710)$ [11] and the $\Lambda(1405)$ [16].

The non-local sink operator used to construct the wave function cannot be used to isolate the state of interest, and hence the standard technique cannot be applied. However it is sufficient to isolate the state at the source using the right eigenvector. Thus, the probability distributions are calculated with each smeared source operator and the right eigenvectors calculated from the standard variational analysis are then applied in order to extract the individual states of interest. 
We find that the right eigenvector alone can successfully isolate the excited states close to the source and indicates that our wave functions should be calculated within 3 time slices after the source.

In summary, the wave function for state $\alpha$ is

$$
\psi^{\alpha}(\vec{p}, t ; \vec{y}, \vec{z}, \vec{w})=\sum_{x} e^{-i \vec{p} \cdot \vec{x}}\left\langle\Omega\left|T\left\{\chi_{1}(\vec{x}, \vec{y}, \vec{z}, \vec{w}) \bar{\chi}_{j}(0)\right\}\right| \Omega\right\rangle u_{j}^{\alpha} .
$$

By averaging over the equally weighted $\{U\}$ and $\left\{U^{*}\right\}$ link configurations, the two-point function is perfectly real and the probability density is proportional to the square of the wave function. In the following we focus on $\vec{p}=0$ and select $t=2$ for the wave functions.

We use $2+1$ flavour $32^{3} \times 64$ configurations provided by the PACS-CS collaboration [19] constructed with the Iwasaki gauge action [17] and the $\mathscr{O}(a)$-improved Wilson action [18] with $\beta=1.90$, giving a lattice spacing of $0.0907(13) \mathrm{fm}$,. The hopping parameters are $0.13727,0.13754$, 0.13770 and 0.13781 giving pion masses of 572, 413, 293 and $156 \mathrm{MeV}$ respectively.

To accurately access the first three states, a $4 \times 4$ variational basis is constructed using the $\chi_{1}$ operator with four smearing levels; 16, 35, 100 and 200 sweeps of Gaussian smearing [20], corresponding to smearing radii of $2.37,3.50,5.92$ and 8.55 lattice units respectively. We fix to Landau gauge by maximizing the $\mathscr{O}\left(a^{2}\right)$-improved fixing functional [21]

$$
\mathscr{F}_{I m p}=\sum_{x, \mu} \operatorname{Re} \operatorname{tr}\left(\frac { 4 } { 3 } \left(U_{\mu}(x)-\frac{1}{12 u_{0}}\left(U_{\mu}(x) U(x+\hat{\mu})+\text { h.c. }\right)\right.\right.
$$

using a Fourier accelerated algorithm [22].

The wave functions observed for all our states show an approximate symmetry over the eight octants surrounding the origin. To improve our statistics we average over these eight octants before presenting the results.

In Fig. 1, an isovolume of the projected ground state wave function at all four quark masses cut in the plane of the $u$ quarks is presented. At all masses, the well-known spherical shape of the ground state is reproduced. Very little mass dependence is seen, consistent with early, quenched wave function studies [23]. Finite volume effects do not appear to be significant in the wave functions of the ground state at any of the hopping parameters used. This is in spite of the fact that the lightest ensemble has $m_{\pi} L=2.23$.

For the Roper wave function in Fig. 2 a $2 S$-like nodal structure is seen at all masses, indicating that the wave function is a radial excitation of the $d$ quark. This observation is also consistent with the predictions of constituent quark models. Even at $m_{\pi}=572 \mathrm{MeV}$, subtle finite volume effects can be seen in the outer shell, with the sides compressed slightly into a non-spherical shape by the boundary. When the quark mass decreases to give $m_{\pi}=413 \mathrm{MeV}$, the radius of the node increases and the finite volume effects become more apparent. In constituent quark models, as the quark mass is reduced, the lighter quarks sit higher in the potential and the range of the spin dependent part is increased. This leads to constituent quarks that sit higher in the potential, and thus have the tail enhanced, leading to an increase in the distance from the centre of mass to the node. This enhancement of the tail, and thus increased finite volume effects are apparent our results.

At $m_{\pi}=293 \mathrm{MeV}$, finite volume effects have become quite severe. This is most likely associated with an increased response from the pion cloud as the quark mass becomes lighter. Even 

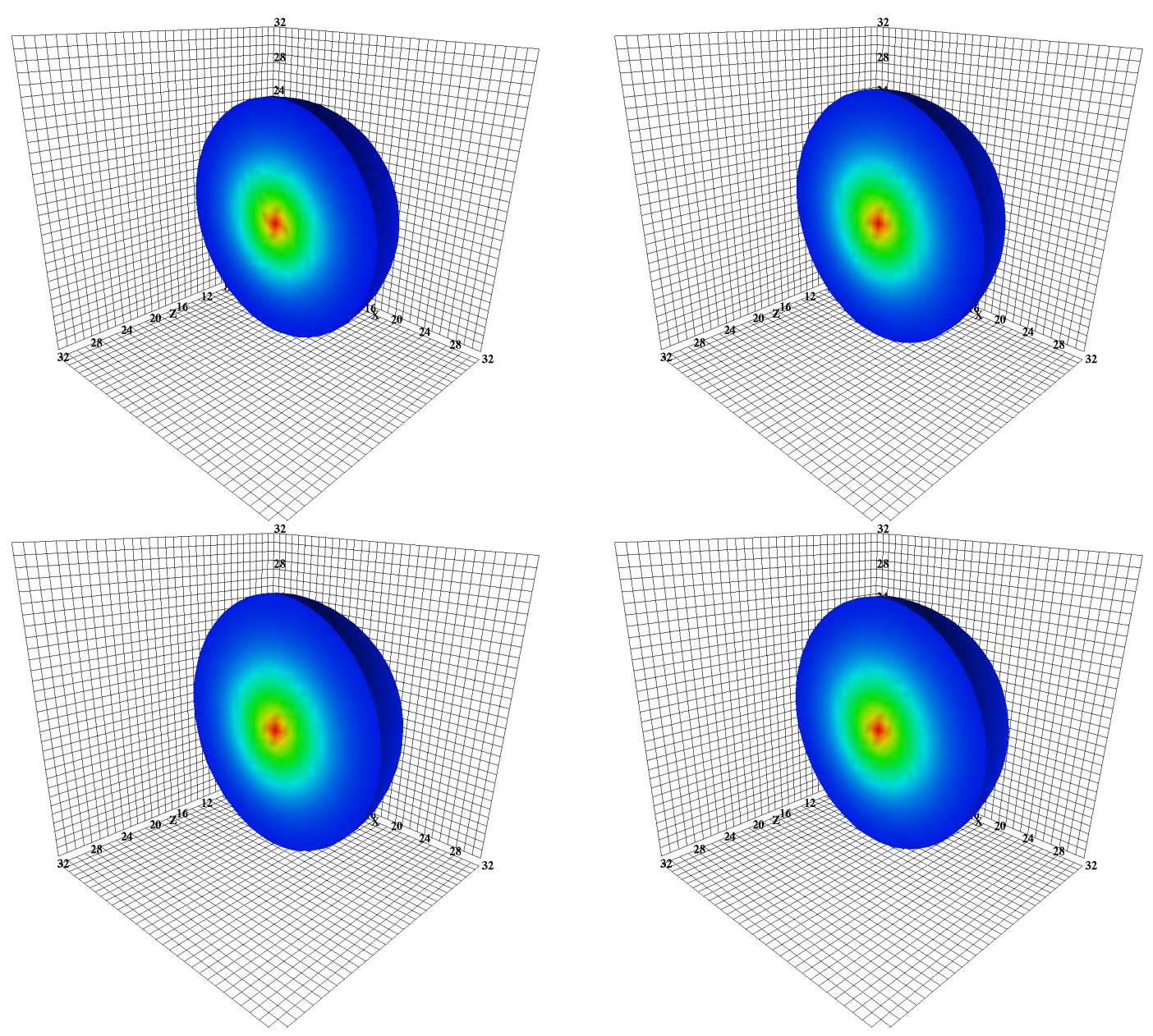

Figure 1: The probability distribution of the ground state $d$ quark about the two $u$ quarks fixed at the centre at each of the four quark masses. Images are ordered from heaviest to lightest quark masses with $\kappa=0.13727$ (top left), $\kappa=0.13754$ (top right), $\kappa=0.13770$ (bottom left) and $\kappa=0.13781$ (bottom right). Very little mass dependence is apparent in the ground state, even at a near-physical quark mass.

with $m_{\pi} L=4.4$, the lattice is too small to fully accommodate the first excited state. At the lightest quark mass, the radius of the node has again increased, and the finite volume effects are even more apparent, with the outer shell being pushed towards a rectangular shape by the boundary.

For the wave function of the second excited state in Fig. 3, a 3S-like nodal structure is observed at all masses. The node is thin at the heavier masses and difficult to see in the rendering. For $m_{\pi}=572 \mathrm{MeV}$, finite volume effects are minimal, and both nodes are spherical in shape and are unaffected by the boundary. Moving to the next lightest quark mass, the nodes move further from the centre of mass, consistent with both quark model predictions and the behaviour of the probability distribution of the Roper. At $m_{\pi}=293 \mathrm{MeV}$, the nodes have again moved further from the centre of the wave function, however, the outer node has taken on a slightly squared-off shape, having been distorted by the boundary of the lattice. Again, this is an indication that, even though the ground state wave function presents as spherical for this quark mass, this excited state is showing clear finite volume effects. Even at relatively modest quark masses, the wave functions of states above 

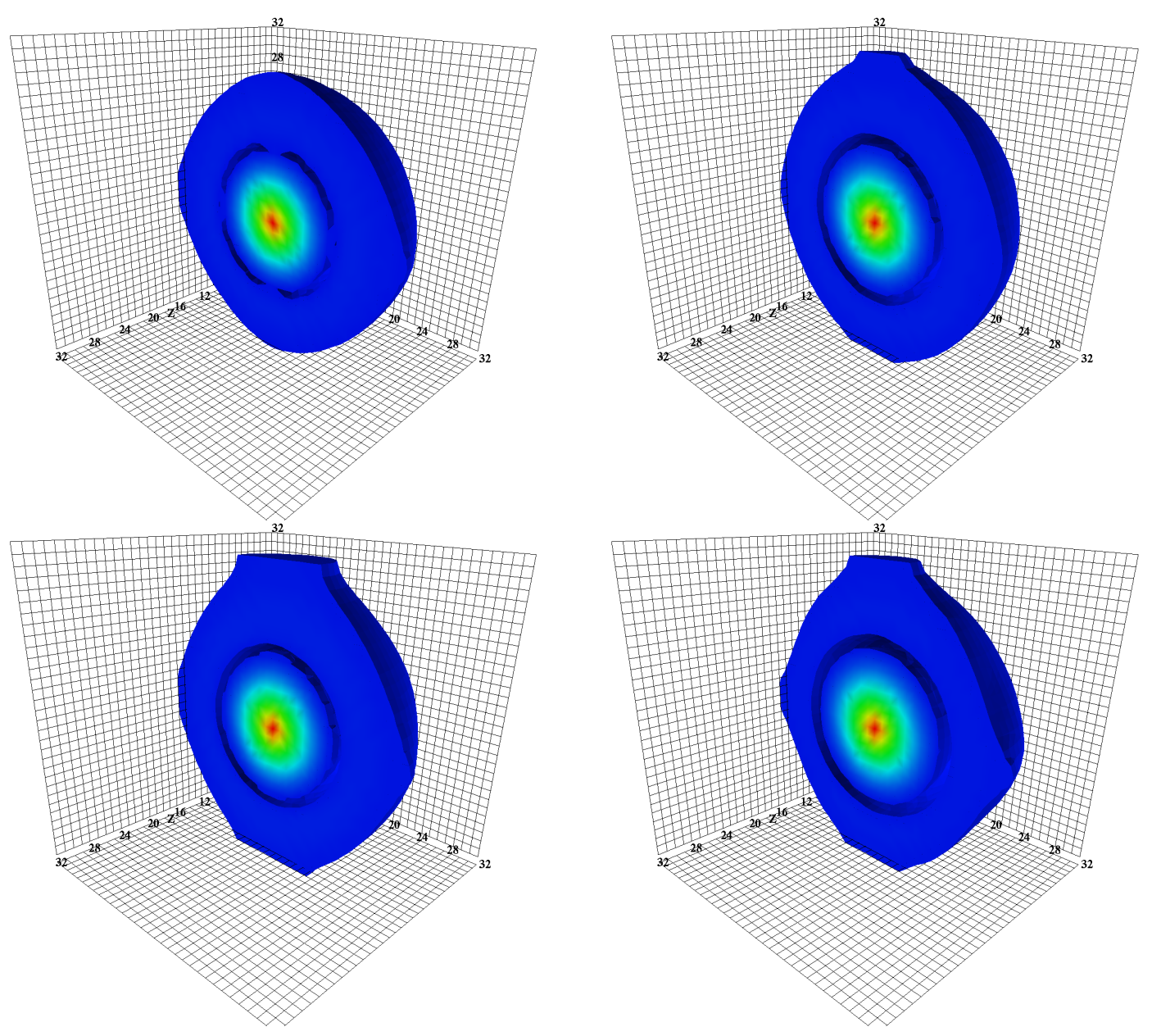

Figure 2: The probability distribution of the $d$ quark in first excited state of the nucleon about the two $u$ quarks fixed at the centre at each of the four quark masses. Images are ordered from heaviest to lightest quark masses with $\kappa=0.13727$ (top left), $\kappa=0.13754$ (top right), $\kappa=0.13770$ (bottom left) and $\kappa=0.13781$ (bottom right). The node in the wave function moves outward as the quark mass decreases, and finite volume effects are seen to increase. The tail of the wave function is enhanced at lower quark masses, likely due to the increased response of the pion cloud.

the decay thresholds show an important relationship with the finite volume of the lattice. The wave function at the near physical quark mass shows even more dramatic finite volume effects, with the outer node resembling a rounded-square shape.

In this study of the mass dependence of the quark probability distribution within excited states of the nucleon, we have shown that both the Roper and the second excited state display the node structure associated with radial excitations of the quarks.

This structure behaves in a manner predicted by early constituent quark models, in that as the quark mass increases, the $d$ quark sits lower in the potential and the nodes are drawn closer to the centre of mass of the probability distribution.

We have also demonstrated that lattices sufficiently large to accomodate the ground state display significant finite volume effects with excited states above the decay threshold as evidenced 

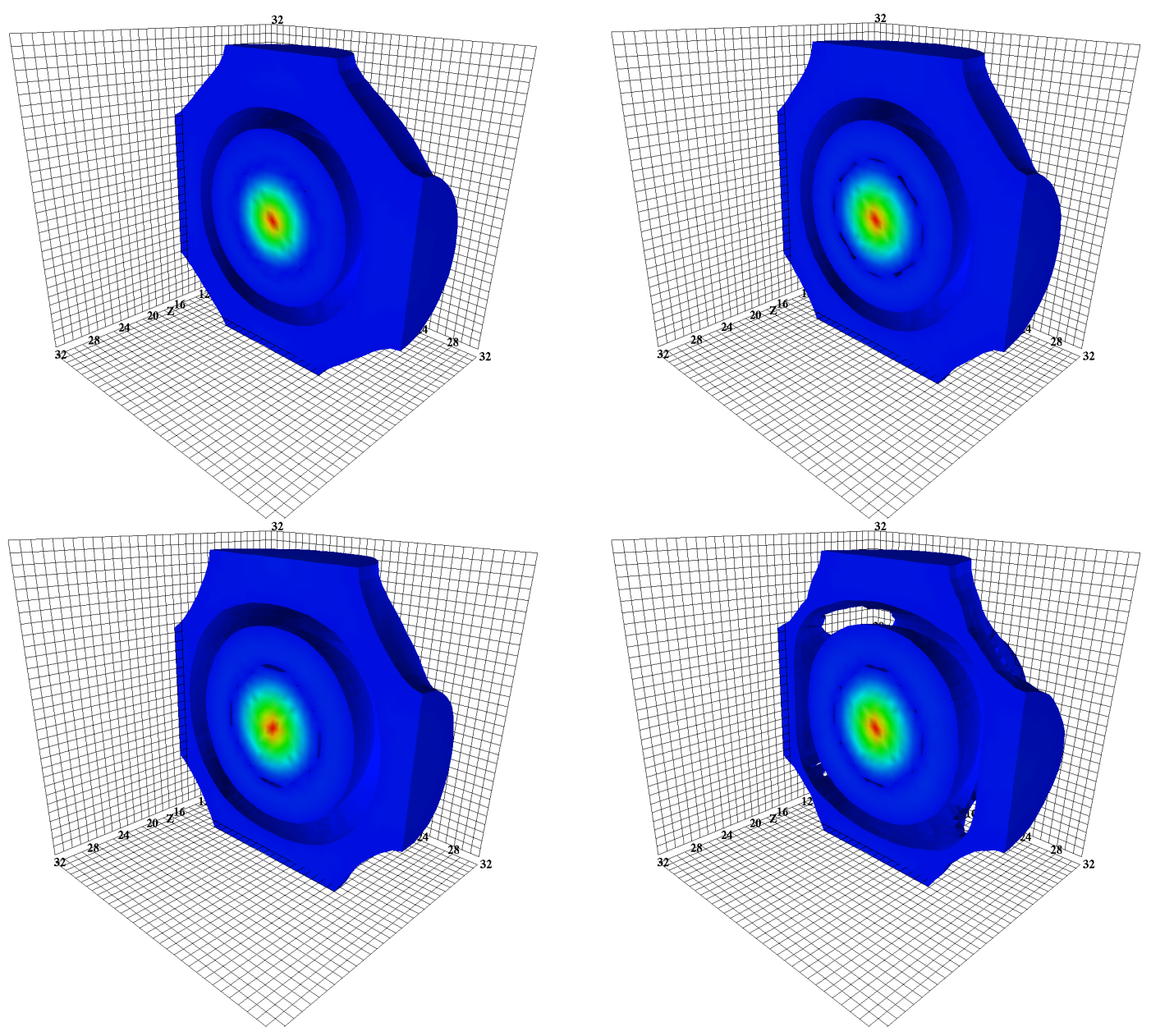

Figure 3: The probability distribution of the $d$ quark in the second even parity excited state of the nucleon about the two $u$ quarks fixed at the centre at each of the four quark masses. Images are ordered from heaviest to lightest quark masses with $\kappa=0.13727$ (top left), $\kappa=0.13754$ (top right), $\kappa=0.13770$ (bottom left) and $\kappa=0.13781$ (bottom right).. Two nodes are evident, consistent with a $3 S$ radial excitation. Important finite volume effects have caused the outermost shell of the wave function to approach shape at lower quark masses.

by the distortion of the probability distributions due to the boundary of the lattice at lighter quark masses. These robust effects are likely associated with the multiparticle components of the excited states.

\section{Acknowledgements}

We thank PACS-CS Collaboration for making their 2+1 flavor gauge-field configurations available and the ILDG for creating the opportunity, tools and formalism for sharing these configurations. This research was undertaken on the NCI National Facility in Canberra, Australia, which is supported by the Australian Commonwealth Government. We also acknowledge eResearch SA for grants of supercomputing time which have enabled this project. This research is supported by the Australian Research Council. 


\section{References}

[1] M. C. Chu, M. Lissia and J. W. Negele, Nucl. Phys. B 360, 31 (1991).

[2] R. Gupta, D. Daniel and J. Grandy, Phys. Rev. D 48, 3330 (1993) [hep-lat/9304009].

[3] D. S. Roberts, P. O. Bowman, W. Kamleh and D. B. Leinweber, Phys. Rev. D 83, 094504 (2011) [arXiv:1011.1975 [hep-lat]].

[4] D. B. Leinweber, Phys. Rev. D 51, 6383 (1995) [nucl-th/9406001].

[5] M. Gockeler et al. [QCDSF and UKQCD and LHPC Collaborations], Phys. Lett. B 532, 63 (2002) [hep-lat/0106022].

[6] S. Sasaki, T. Blum and S. Ohta, Phys. Rev. D 65, 074503 (2002) [hep-lat/0102010].

[7] W. Melnitchouk et al., Phys. Rev. D 67, 114506 (2003) [hep-lat/0202022].

[8] F. X. Lee, S. J. Dong, T. Draper, I. Horvath, K. F. Liu, N. Mathur and J. B. Zhang, Nucl. Phys. Proc. Suppl. 119, 296 (2003) [hep-lat/0208070].

[9] S. Basak et al., Phys. Rev. D 76, 074504 (2007) [arXiv:0709.0008 [hep-lat]].

[10] J. Bulava, R. G. Edwards, E. Engelson, B. Joo, H-W. Lin, C. Morningstar, D. G. Richards and S. J. Wallace, Phys. Rev. D 82, 014507 (2010) [arXiv:1004.5072 [hep-lat]].

[11] M. S. Mahbub et al. [CSSM Lattice Collaboration], Phys. Lett. B 707, 389 (2012) [arXiv:1011.5724 [hep-lat]].

[12] C. Michael, Nucl. Phys. B 259, 58 (1985).

[13] M. Luscher and U. Wolff, Nucl. Phys. B 339, 222 (1990).

[14] M. S. Mahbub, A. O.Cais, W. Kamleh, B. G. Lasscock, D. B. Leinweber and A. G. Williams, Phys. Rev. D 80, 054507 (2009) [arXiv:0905.3616 [hep-lat]].

[15] L. D. Roper, Phys. Rev. Lett. 12, 340 (1964).

[16] B. J. Menadue, W. Kamleh, D. B. Leinweber and M. S. Mahbub, Phys. Rev. Lett. 108, 112001 (2012) [arXiv:1109.6716 [hep-lat]].

[17] Y. Iwasaki, arXiv:1111.7054 [hep-lat].

[18] B. Sheikholeslami and R. Wohlert, Nucl. Phys. B 259, 572 (1985).

[19] S. Aoki et al. [PACS-CS Collaboration], Phys. Rev. D 79, 034503 (2009) [arXiv:0807.1661 [hep-lat]].

[20] S. Gusken, Nucl. Phys. Proc. Suppl. 17, 361 (1990).

[21] F. D. R. Bonnet, P. O. Bowman, D. B. Leinweber, A. G. Williams and D. G. Richards, Austral. J. Phys. 52, 939 (1999) [hep-lat/9905006].

[22] C. T. H. Davies et al. Phys. Rev. D 37, 1581 (1988).

[23] M. W. Hecht and T. A. DeGrand, Phys. Rev. D 46, 2155 (1992). 